\title{
High-risk lesions diagnosed at MRI-guided vacuum-assisted breast biopsy: can underestimation be predicted?
}

\author{
Pavel Crystal • Arifa Sadaf • Karina Bukhanov • \\ David McCready • Frances O'Malley • \\ Thomas H. Helbich
}

Received: 5 June 2010 /Revised: 25 July 2010 / Accepted: 30 July 2010 / Published online: 14 September 2010

(C) European Society of Radiology 2010

\begin{abstract}
Objectives To evaluate the frequency of diagnosis of highrisk lesions at MRI-guided vacuum-assisted breast biopsy (MRgVABB) and to determine whether underestimation may be predicted.

Methods Retrospective review of the medical records of 161 patients who underwent MRgVABB was performed. The underestimation rate was defined as an upgrade of a high-risk lesion at MRgVABB to malignancy at surgery. Clinical data, MRI features of the biopsied lesions, and histological diagnosis of cases with and those without underestimation were compared.

Results Of 161 MRgVABB, histology revealed 31 (19\%) high-risk lesions. Of 26 excised high-risk lesions, 13 (50\%) were upgraded to malignancy. The underestimation rates of lobular neoplasia, atypical apocrine metaplasia, atypical ductal hyperplasia, and flat epithelial atypia were 50\%
\end{abstract}

P. Crystal • A. Sadaf · K. Bukhanov • T. H. Helbich

Division of Breast Imaging, Mount Sinai Hospital and University

Health Network,

600 University Avenue,

M5G 1X5 Toronto, ON, Canada

D. McCready

Department of Surgical Oncology, Princess Margaret Hospital, 610 University Avenue,

M5G 2M9 Toronto, ON, Canada

\section{F. O'Malley}

Department of Pathology and Laboratory Medicine,

Mount Sinai Hospital,

600 University Avenue,

M5G 1X5 Toronto, ON, Canada
(4/8), $100 \%(5 / 5), 50 \%(3 / 6)$ and 50\% (1/2) respectively. There was no underestimation in the cases of benign papilloma without atypia (0/3), and radial scar $(0 / 2)$. No statistically significant differences $(p>0.1)$ between the cases with and those without underestimation were seen in patient age, indications for breast MRI, size of lesion on MRI, morphological and kinetic features of biopsied lesions.

Conclusions Imaging and clinical features cannot be used reliably to predict underestimation at MRgVABB. All highrisk lesions diagnosed at MRgVABB require surgical excision.

Keywords Vacuum-assisted breast biopsy · Breast MRI . MRI-guided breast biopsy . High-risk breast lesions . Underestimation

P. Crystal $(\bowtie)$

Mount Sinai Hospital,

Room 574, 600 University Avenue,

Toronto, ON M5G 1X5, Canada

e-mail: pavel.crystal@uhn.on.ca

Present Address:

T. H. Helbich

Department of Radiology, Division of Molecular and Gender Imaging, Medical University of Vienna, General Hospital Vienna, Waehringer Guertel 18-20, Floor 7f,

1090 Vienna, Austria

Present Address:

F. O'Malley

Department of Laboratory Medicine,

University of Toronto, St. Michael's Hospital,

30 Bond St.,

Toronto, ON M5B 1W8, Canada

e-mail: omalleyf@smh.ca 


\section{Introduction}

Magnetic resonance imaging (MRI) has emerged as the most sensitive technique for the screening, detection and diagnosis of breast cancer [1-3]. Moreover, MRI of the breast is superior not only for diagnosis of invasive carcinoma but also for detection of ductal carcinoma in situ (DCIS) and even premalignant breast lesions [4, 5]. MRI-guided vacuumassisted breast biopsy (MRgVABB) is a safe and accurate technique for evaluation of otherwise occult MRI-detected breast lesions [6-12]. However, a considerable proportion of lesions diagnosed on percutaneous breast biopsies are classified as high-risk lesions [13]. The high-risk histology includes atypical ductal hyperplasia (ADH), lobular neoplasia (LN) (regrouping of the former atypical lobular hyperplasia (ALH) and lobular carcinoma in situ (LCIS)), flat epithelial atypia (FEA), papillary lesions, radial scar/complex sclerosing lesion, phyllodes tumour, and mucocele-like lesions [1419]. When these lesions are diagnosed at image-guided biopsy, the presence of an underlying malignancy may be underestimated, yielding a challenge in clinical management [20-22]. The management of high-risk lesions diagnosed on stereotactic or ultrasound-guided VABB has been very well researched [23-28] and surgical excision is usually recommended. There are scanty data regarding underestimation rate at $\mathrm{MRgVABB}$ and management of high-risk lesions diagnosed at MRgVABB [11, 12, 29].

Thus the purpose of our study was to determine the frequency of diagnosis of high risk lesions at MRgVABB, to assess the rate of underestimation as compared to subsequent surgical excision, and to determine whether clinical, imaging, or histological features of high-risk lesions diagnosed at MRgVABB can be used to predict upgrading to malignancy at subsequent surgical excision.

\section{Materials and methods}

In a protocol approved by our institutional research ethics board, we retrospectively reviewed the medical records and imaging studies of 161 patients who underwent MRgVABB during a 36-month period, between March 2006 and March 2009. MRgVABB histology yielded malignancy in 19 $(12 \%)$ out of 161 lesions that underwent MRI biopsy during the study period, of which 10 were invasive cancer and nine were DCIS. MRgVABB yielded benign histological findings in $111(69 \%)$ and high-risk findings in 31 (19\% of all lesions). Surgical excision, recommended for all 31 high-risk lesions, was performed on 26 lesions and these cases constituted the basis of this study.

Five women with high risk lesions at MRgVABB declined excisional biopsy, namely two women with ALH, two women with papilloma, and one woman with ADH. These five high- risk lesions remained stable or diminished in size at follow-up MRI examinations. Mean time of MRI follow-up for nonexcised high risk lesions was 14 months, range 6-26 months. Clinical and mammographic follow-up in these five women was also unremarkable.

\section{MRI indications and evaluations}

All screening and diagnostic MRI studies were performed at our institution. The indications for breast MRI included highrisk screening (e.g. in women with a genetic predisposition, a strong family history of breast cancer, previous breast cancer, biopsy-proven diagnosis of atypical hyperplasia or lobular carcinoma in situ, or previous mantle irradiation for Hodgkin's disease), extent of disease assessment in the ipsilateral or contralateral breast in a woman with biopsy-proven breast cancer, follow-up after previous breast MRI, or problemsolving. If an ultrasound correlate for the MRI-detected lesion is confidently identified, biopsy is usually performed under ultrasound guidance. For MRI-suspected lesions that have no ultrasound correlates, that have vague ultrasound findings that definitely do not correlate with the MRI lesion, or that are better seen on MRI than on ultrasound, biopsy is usually performed under MRI guidance.

Biopsy radiologists and technique

All biopsies during the study period were performed with a 9-gauge MRI-compatible vacuum-assisted biopsy device (Automated Tissue Excision and Collection, Suros Surgical Systems, Hologic, Inc., Indianapolis, IN, USA) under sterile conditions. All biopsies were performed by four radiologists with 5-15 years' experience in breast imaging All biopsy radiologists had experience interpreting breast MRI studies and performing percutaneous breast biopsy under stereotactic or ultrasound guidance. The results we present include our learning experience. Biopsies were performed with the patient positioned prone in a $1.5 \mathrm{~T}$ MRI unit (Signa, GE Medical Systems, Milwaukee, WI, USA). A dedicated eight-channel breast coil (Sentinelle Vanguard Breast MR Auxiliary Table, Sentinelle Medical, Toronto, Canada) was used. Multiple contiguous samples were obtained at $360^{\circ}$ with at least one clockwise rotation. The biopsy site was marked with a titanium marker (Trimark, Suros) at the discretion of the radiologist performing the biopsy. After biopsy, the breast was compressed; sterile strips and a sterile gauze bandage were applied. When a clip was placed, a two-view digital mammogram was obtained to document clip placement (6-9).

\section{Pathology methods and definitions}

Pathological evaluation of MRgVABB specimens was performed by three breast pathologists with 6-14 years' 
experience in interpretation of percutaneous core-needle breast biopsies. Specimens were fixed in neutral buffered formalin. The tissue sampled was entirely embedded and examined. For all the patients, results of MRI-guided biopsies were reviewed during a multidisciplinary session to assess radiological-pathological concordance. Excisional biopsy was recommended for all high-risk lesions; namely for atypical ductal hyperplasia (ADH), lobular neoplasia (LN) (regrouping of the former atypical lobular hyperplasia (ALH) and lobular carcinoma in situ (LCIS)), atypical apocrine proliferation, papillary lesions, radial scar/complex sclerosing lesion and flat epithelial atypia (FEA). Excisional biopsy was performed after preoperative needle-localisation using the titanium clip as a landmark for localisation. Excisional biopsies were performed by two breast surgeons, each with more than 15 years' experience.

At histopathology underestimation was defined as an upgrade of the high-risk lesion diagnosed at MRgVABB to ductal carcinoma in situ (DCIS) or invasive carcinoma diagnosed at surgery. The high-risk lesion underestimation rate was defined as the quotient of these upgraded lesions and all excised high-risk lesions diagnosed at $\operatorname{MRgVABB}[24,26]$.

\section{Data collection and statistical analysis}

Data collected included clinical information (clinical history, patient age, indication for breast MRI); MRI features of the biopsied lesions (size, location, morphological and kinetic characteristics according to the BI-RADS lexicon [30]); procedure-related variables (distance from the tip of biopsy device to the target, if any, location of post-biopsy haematoma, complications and previous experience of the biopsy radiologist); and histological results. Biopsies were considered technically successful based on the distance $\leq 3$ millimetres between the needle and the lesion margin and the presence of post-biopsy haematoma at the edge of the lesion's location.

Data were entered in a spread-sheet program (Excel, Microsoft). Chi-squared, T-test, and Fisher's exact tests for statistical significance were performed with on-line statistical software (simple interactive statistical analysis at home.clara. net/sisa), and $p<0.05$ was considered significant.

\section{Results}

Patients, indications for MRI, and lesions

The 26 high-risk lesions diagnosed at MRI-guided 9-gauge vacuum-assisted breast biopsy that were subsequently surgically excised occurred in 25 women with a mean age of 54 years (range, 37-73 years).
The indications for breast MRI that led to detection of these 26 high-risk lesions included screening of high-risk women in $10(38 \%)$, ipsilateral surveillance in women with a history of breast carcinoma in 7 (27\%), contralateral surveillance in women with a history of breast carcinoma in three $(12 \%)$, problem solving in $4(15 \%)$, preoperative screening of the contralateral breast in $1(4 \%)$, and preoperative evaluation of disease extent in the ipsilateral breast in one $(4 \%)$ of the cases.

The median size of the 26 high-risk lesions included was $14 \mathrm{~mm}$, (range, 5-60 mm).

The MRI morphological features of the 26 high-risk lesions included $8(31 \%)$ masses and 18 (69\%) non-masslike enhancements. The MRI kinetic features of the 26 high-risk lesions included 14 (54\%) persistent, 10 (38\%) plateau, and $2(8 \%)$ washout patterns of enhancement.

All biopsies were considered technically successful based on pre-biopsy needle position and location of postbiopsy haematoma. No complications were encountered.

\section{Surgical histology}

Of the 26 excised high-risk lesions, surgical histological examination yielded invasive mammary carcinoma in two $(8 \%)$ of the lesions (one case of invasive ductal carcinoma and one case of mixed invasive ductal and invasive lobular carcinoma); DCIS in 11 (42\%) of the lesions; and high-risk pathological features in $13(50 \%)$ of the lesions (Table 1). Thus the underestimation rate for all lesions was 50\% (13 out of 26). The underestimation rates of atypical apocrine proliferation, lobular neoplasia, $\mathrm{ADH}$, and flat epithelial atypia were $100 \%$ ( 5 out of 5 ), $50 \%$ (4 out of 8 ), $50 \%$ (3 out of 6 ) and $50 \%$ ( 1 out of 2 ), respectively. There was no underestimation in the cases of papillary lesions without atypia ( 0 out of 3 ) and radial scar ( 0 out of 2$)$.

The underestimation rates based on indications for breast MRI are presented in Table 2. There were no statistical differences between the compared groups although there was a trend $(p>0.05)$ towards a higher $(70 \%, 7 / 10)$ upgrade rate in women with a personal history of breast carcinoma versus a $30 \%(3 / 10)$ underestimation rate in women who had high-risk lesions detected at screening MRI.

Comparative features of the high-risk lesions that were upgraded to malignancy and high-risk lesions that were confirmed to be benign or high-risk after excision are presented in Table 3. No significant difference was found in the likelihood of high-risk lesion underestimation as a function of patient age, lesion size, morphological lesion type and pattern of lesion enhancement. There was a nonsignificant trend $(p>0.08)$ towards larger MRI-measured lesion size in the underestimated cases (mean size $24.3 \pm$ $21.5 \mathrm{~mm}$ ) versus those without underestimation (mean size $12.7 \pm 7.9 \mathrm{~mm})$. 
Table 1 Correlation of histology results after MRgVABB (MRI-guided vacuum-assisted breast biopsy) with final pathological result after excisional biopsy

\begin{tabular}{|c|c|c|c|c|c|c|c|c|}
\hline \multirow[t]{2}{*}{ MRgVABB } & \multicolumn{8}{|c|}{ Surgical excision } \\
\hline & Invasive $\mathrm{Ca}$ & DCIS & $\mathrm{ADH}$ & Lobular neoplasia & Papillary lesion & Radial scar & Flat atypia & Total \\
\hline $\mathrm{ADH}$ & 1 & 2 & 2 & & & & 1 & 6 \\
\hline Lobular neoplasia & 1 & 3 & & 4 & & & & 8 \\
\hline Atypical apocrine proliferation & & 5 & & & & & & 5 \\
\hline Flat epithelial atypia & & 1 & 1 & & & & & 2 \\
\hline Benign Papillary lesions & & & 1 & 1 & 1 & & & 3 \\
\hline Radial scar & & & & & & 2 & & 2 \\
\hline Total & 2 & 11 & 4 & 5 & 1 & 2 & 1 & 26 \\
\hline
\end{tabular}

$A D H$ Atypical Ductal Hyperplasia, DCIS Ductal Carcinoma in Situ

Note.-Data are numbers of lesions.

\section{Discussion}

The results of our study indicate that imaging and clinical features cannot be used reliably to predict underestimation at MRgVABB. The diagnosis of high-risk lesion at MRgVABB warrants surgical excision because of the very high $(50 \%)$ frequency of underestimation of breast carcinoma. Furthermore, the underestimation rate in the subgroup of high-risk lesions with atypia $(\mathrm{ADH}, \mathrm{LN}$, atypical apocrine proliferation, and FEA) was even higher and reached 62\% (13 out of 21 ) in our small study. We did not find underestimation in the cases of benign papilloma and radial scar diagnosed at MRgVABB and thus additional data are required regarding the management of these lesions.

MRgVABB revealed high-risk lesions in 19\% (31 out of $161)$ of the biopsied lesions. Both frequency of high-risk lesions at MRgVABB and underestimation rates in our study are higher than previously reported in other studies (Table 4) evaluating outcomes of MR-guided breast biopsy $[11,12,29,31,32]$. This might be explained by the high pretest probability for breast cancer in our study population, which in addition to asymptomatic women undergoing screening MRI included a substantial proportion of diagnostic cases and patients with a personal history of breast carcinoma. Another factor that could have contributed to the high underestimation rate is MRI features of the target lesions that were usually small masses or non-contiguous, non-mass-enhancing lesions.

MRgVABB revealed lobular neoplasia in 6\% (10 out of 161 cases) and atypical apocrine proliferation in 3\% (5 out of 161 cases) and the upgrade rates to malignancy were very high for these entities (50\% and $100 \%$ respectively). These are novel findings. It may be related to both lesion biology and/or MRI features. Lobular neoplasia is not usually seen at mammography and is commonly considered to be an incidental finding at stereotactic biopsy which is performed for suspect microcalcifications [33]. In fact, the previously reported rate of lobular neoplasia on stereotactic breast biopsy is only $1 \%$, while upgrade to breast cancer occurs in approximately $20 \%[33,34]$. Lobular neoplasia is considered to be not only a cancer precursor but also a risk factor for bilateral breast cancer and commonly presents

Table 2 The underestimation rates of high-risk lesions based on indications for breast MRI

\begin{tabular}{lccc}
\hline Indications for breast MRI & Total number of cases & Refused surgery & Underestimation rate \\
\hline Screening & 13 & 3 & $30 \%(3 / 10)$ \\
Ipsilateral preoperative staging & 1 & 0 & $100 \%(1 / 1)$ \\
Contralateral preoperative screening & 2 & 1 & $0 \%(0 / 1)$ \\
Ipsilateral surveillance in women with previous breast carcinoma & 4 & 0 & $75 \%(3 / 4)$ \\
Contralateral surveillance in women with previous breast carcinoma & 7 & 1 & $67 \%(4 / 6)$ \\
Problem solving & 4 & 0 & $50 \%(2 / 4)$ \\
Total & 31 & 5 & $50 \%(13 / 26)$ \\
\hline
\end{tabular}


Table 3 Possible predictors of underestimation in 24 surgically excised lesions with diagnosis of high-risk lesion at MR-guided 9-gauge vacuumassisted breast biopsy

\begin{tabular}{|c|c|c|c|}
\hline Variables & Cases with underestimation $(n=13)$ & Cases without underestimation $(n=13)$ & $\mathrm{P}$ value \\
\hline Mean age, years & 52 & 54 & $p>0.5$ \\
\hline Mean lesion size, $\mathrm{mm}$ and standard deviation (SD) & $24.3(21.5)$ & $12.7(7.9)$ & $p>0.08$ \\
\hline Number of lesions with mass enhancement & 4 & 4 & $p>0.5$ \\
\hline Number of lesions without mass enhancement & 9 & 9 & $p>0.5$ \\
\hline Wash-out enhancement & 1 & 1 & $p>0.5$ \\
\hline Plateau enhancement & 6 & 4 & $p>0.5$ \\
\hline Progressive enhancement & 6 & 8 & $p>0.5$ \\
\hline
\end{tabular}

pathologically as multiple small foci occupying a large area of breast. Therefore, MRI may detect only a small part of the pathological process in cases of lobular neoplasia and this may explain the high upgrade rate when a larger area is surgically excised. Thus, an example of underestimation of lobular neoplasia with upgrade to DCIS is presented in Fig. 1.

Our result of $50 \%$ (3 out of 6) $\mathrm{ADH}$ underestimation rate is similar to the $\mathrm{ADH}$ underestimation reported by Liberman et al [29]. All cases of atypical apocrine proliferation diagnosed at MRgVABB were underestimated in our study. We hypothesise that this might be explained by extreme difficulty in differentiating this entity from DCIS on pathological evaluation of core biopsy specimens.

Controversy persists regarding the need for excision of benign concordant papillomas and concordant radial scars diagnosed at image-guided vacuum-assisted biopsy. Our number of cases is extremely small and should not influence existing policies, however if lack of upgrade in these entities persists an option of MRI follow-up may be justified rather than excision.

We demonstrated that high-risk lesions diagnosed on MRgVABB in women who underwent breast MRI for clinical indications other than screening are more likely to be malignant at excisional biopsy than high-risk lesions diagnosed in patients who underwent breast MRI for screening purposes (Table 2). Our results corroborate earlier studies that attempted to evaluate patients' characteristics in order to better define predictive factors for malignancy in breast MRI lesions. Thus, Han et al [11] found the probability of malignancy to be higher in the diagnostic breast MRI setting compared with screening MRI (36\% vs. 14\%, respectively) in their evaluation of MRI-guided breast biopsy outcomes. Two other studies $[35,36]$ demonstrated a higher frequency of malignancy in lesions identified by MRI for the clinical indication of newly diagnosed cancer versus other indications.

We have not identified that underestimation of malignancy at MRgVABB increases significantly with increasing lesion size on imaging and this is similar to results of Liberman et al. [29] although somehow unexpected as it would be reasonable to assume that the larger the target (the MRI lesion), the relatively more limited the sampling. In fact, Houssami et al. [37] report a highly significant association between lesion size on imaging and underestimation of malignancy on percutaneous stereotactic and ultrasound-guided image-guided breast biopsy in their large series of 758 surgically excised lesions that were diagnosed as high-risk on core biopsy. Larger series of MRgVABB are

Table 4 Comparison of previously reported rates of high-risk lesions diagnosed at MRI-guided vacuum-assisted breast biopsy and malignancy underestimation rates

\begin{tabular}{lllcr}
\hline Author/year & High-risk entity & Needle size, G (gauge) & Rate of high-risk lesions (\%) & Underestimation rate, \% \\
\hline Liberman et al. (2007) [29] & $\mathrm{ADH}^{\mathrm{a}}$ & $9 \mathrm{G}$ & $6 \%(15 / 237)$ & $38 \%(5 / 13)$ \\
Han et al. (2008) [11] & $\mathrm{Mix}^{\mathrm{b}}$ & $9 \mathrm{G}$ & $14 \%(21 / 150)$ & $25 \%(4 / 16)$ \\
Friedman et al. (2009) [12] & $\mathrm{Mix}^{\mathrm{b}}$ & $10 \mathrm{G}$ & $14 \%(28 / 197)$ & Not available \\
Noroozian et al. (2010) [31] & $\mathrm{Mix}^{\mathrm{b}}$ & $9 \mathrm{G}$ & $9 \%(7 / 75)$ & $17 \%(1 / 6)$ \\
Malhaire et al. (2010) [32] & Mix $^{\mathrm{b}}$ & $10 \mathrm{G}$ & $14 \%(10 / 72)$ & $13 \%(1 / 8)$ \\
Present study & Mix $^{\mathrm{b}}$ & $9 \mathrm{G}$ & $19 \%(31 / 161)$ & $50 \%(13 / 26)$ \\
\hline
\end{tabular}

a $(\mathrm{ADH})=$ Atypical ductal hyperplasia

b Includes ADH, lobular neoplasia, atypical apocrine proliferation, flat epithelial atypia, papillary lesions, and radial scar 

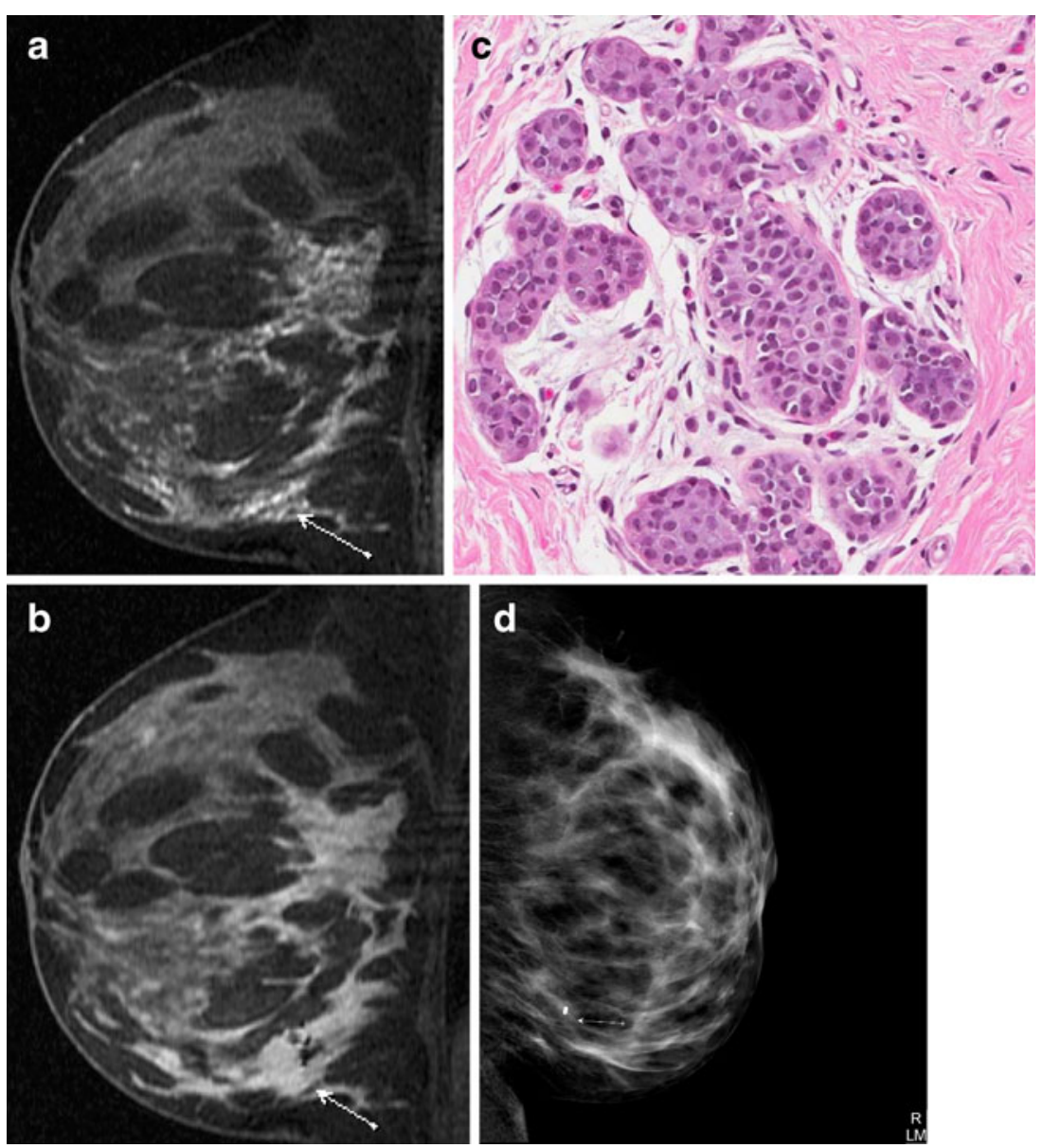

Fig. 1 a A 40-year-old woman with a history of a left lumpectomy for carcinoma three years before underwent routine surveillance MRI examination. Sagittal image from a T1-weighted 3D fat-suppressed fast spoiled gradient-echo sequence after IV administration of gadolinium shows an area of progressive clumped enhancement (arrow) in ductal distribution at the 6 o'clock position. b Sagittal image from post-contrast T1-weighted 3D fat-suppressed fast spoiled gradient-echo sequence immediately after biopsy. Post-biopsy haematoma (arrow) obscures the biopsied area of clumped ductal enhancement. c Pathological evaluation of MR-guided biopsy specimen

required in order to further confirm the lack of association between lesion size and malignancy underestimation observed by us at MRgVABB.

We did not identify that dynamic and morphological parameters predict the underestimation rate of high-risk lesions diagnosed at MRgVABB. These results support previously published studies [11,29] that did not find an association between the morphology and kinetics features of lesions undergoing MRgVABB and malignancy underestimation.

Our study has several limitations. First, the number of lesions is small. However, our results contribute to the growing body of clinical evidence evaluating high-risk lesions at breast MRI. Second, there is a potential bias related to the different experiences of radiologists who revealed lobular neoplasia (LN). High-power microphotograph of lobular neoplasia (LN) involving a terminal duct lobular unit. The arrow highlights the characteristic cells of LN. These cells have round nuclei and intracytoplasmic vacuoles. d Right lateral view before preoperative needle-localisation. There is no mammographic abnormality in the area of the clip (arrow) that was placed at the time of MR-guided vacuum-assisted biopsy. Excisional biopsy was performed after preoperative mammographically-guided needle-localisation and revealed a $16-\mathrm{mm}$ high-grade DCIS

performed biopsies. Third, there was no retrospective pathological review, resulting in the potential for variability in the diagnosis of atypical lesions at MRgVABB. In this regard, it had been shown previously that strict adherence to the diagnostic criteria allows excellent agreement to be achieved in the diagnosis of high-risk lesions [38].

In conclusion, our data indicate that the diagnosis of high-risk lesions at MRgVABB warrants surgical excision because of the very high frequency of underestimation of breast carcinoma. Additional data are required regarding the management of benign papilloma and radial scar diagnosed at MRgVABB. Future research should explore the use of a combination of patient characteristics, imaging (target lesion) and histopathological features on MRgVABB that 
are predictive of excision histology outcome (the likelihood of malignancy) to identify women who should be managed surgically and possibly to identify those who may be able to avoid surgery after diagnosis of high-risk lesions at MRgVABB histology. Until such evidence exists, the standard management of all high-risk lesions diagnosed at MRgVABB is excisional surgical biopsy.

Acknowledgements We gratefully acknowledge Dr. Marina and Dr. Brot Andrade's contribution to the pathology review of the study cases.

This research was partially supported by Patricia and Allan Friedland of the Finkler-Friedland Family Foundation.

\section{References}

1. Saslow D, Boetes C, Burke W, Harms S, Leach MO, Lehman CD, Morris E, Pisano E, Schnall M, Sener S, Smith RA, Warner E, Yaffe M, Andrews KS, Russell CA (2007) American Cancer Society guidelines for breast screening with MRI as an adjunct to mammography. CA Cancer J Clin 57:75-89, Erratum in: CA Cancer J Clin 57:185

2. Miller JC, Rafferty EA, Specht MC, Thrall JH, Lee SI (2008) When is breast magnetic resonance imaging recommended for cancer detection? J Am Coll Radiol 5:224-226

3. Granader EJ, Dwamena B, Carlos RC (2008) MRI and mammography surveillance of women at increased risk for breast cancer: recommendations using an evidence-based approach. Acad Radiol 15:1590-1595

4. Kuhl CK, Schrading S, Bieling HB, Wardelmann E, Leutner CC, Koenig R, Kuhn W, Schild HH (2007) MRI for diagnosis of pure ductal carcinoma in situ: a prospective observational study. Lancet 370:485-492

5. Riedl CC, Ponhold L, Flöry D, Weber M, Kroiss R, Wagner T, Fuchsjäger M, Helbich TH (2007) Magnetic resonance imaging of the breast improves detection of invasive cancer, preinvasive cancer, and premalignant lesions during surveillance of women at high risk for breast cancer. Clin Cancer Res 13:6144- 6152

6. Liberman L, Bracero N, Morris E, Thornton C, Dershaw DD (2005) MRI-guided 9-gauge vacuum-assisted breast biopsy: initial clinical experience. AJR Am J Roentgenol 185:183-193

7. Lehman CD, Deperi ER, Peacock S, McDonough MD, Demartini WB, Shook J (2005) Clinical experience with MRI-guided vacuum-assisted breast biopsy. AJR Am J Roentgenol 184:1782-1787

8. Floery D, Helbich TH (2006) MRI-Guided percutaneous biopsy of breast lesions: materials, techniques, success rates, and management in patients with suspected radiologic-pathologic mismatch. Magn Reson Imaging Clin N Am 14:411-425

9. Orel SG, Rosen M, Mies C, Schnall MD (2006) MR imagingguided 9-gauge vacuum-assisted core-needle breast biopsy: initial experience. Radiology 238:54-61

10. Perlet C, Heywang-Kobrunner SH, Heinig A, Sittek H, Casselman J, Anderson I, Taourel P (2006) Magnetic resonance-guided, vacuum-assisted breast biopsy: results from a European multicenter study of 538 lesions. Cancer 106:982-990

11. Han BK, Schnall MD, Orel SG, Rosen M (2008) Outcome of MRI-guided breast biopsy. AJR Am J Roentgenol 191:1798-1804

12. Friedman P, Enis S, Pinyard J (2009) Magnetic resonance imagingguided vacuum-assisted breast biopsy: an initial experience in a community hospital. Can Assoc Radiol J 60:196-200
13. Houssami N, Ciatto S, Bilous M, Vezzosi V, Bianchi S (2007) Borderline breast core needle histology: predictive values for malignancy in lesions of uncertain malignant potential (B3). Br J Cancer 96:1253-1257

14. Liberman L (2000) Centennial dissertation. Percutaneous imagingguided core breast biopsy: state of the art at the millennium. AJR Am J Roentgenol 174:1191-1199

15. Sewell CW (2004) Pathology of high-risk breast lesions and ductal carcinoma in situ. Radiol Clin North Am 42:821-830

16. Wallis M, Tardivon A, Helbich T, Schreer I (2007) Guidelines from the European Society of Breast Imaging for diagnostic interventional breast procedures. Eur Radiol 17:581-588

17. Costa A, Zanini V (2008) Precancerous lesions of the breast. Nat Clin Pract Oncol 5:700-704

18. Johnson NB, Collins LC (2009) Update on percutaneous needle biopsy of nonmalignant breast lesions. Adv Anat Pathol 16:183195

19. Chivukula M, Bhargava R, Tseng G, Dabbs DJ (2009) Clinicopathologic implications of "flat epithelial atypia" in core needle biopsy specimens of the breast. Am J Clin Pathol 131:802-808

20. Liberman L (2000) Clinical management issues in percutaneous core breast biopsy. Radiol Clin North Am 38:791-807

21. Jacobs TW, Connolly JL, Schnitt SJ (2002) Nonmalignant lesions in breast core needle biopsies: to excise or not to excise? Am J Surg Pathol 26:1095-1110

22. Lawton TJ, Georgian-Smith D (2009) Excision of high-risk breast lesions on needle biopsy: is there a standard of core? AJR Am J Roentgenol 192:W268

23. Jackman RJ, Birdwell RL, Ikeda DM (2002) Atypical ductal hyperplasia: can some lesions be defined as probably benign after stereotactic 11-gauge vacuum-assisted biopsy, eliminating the recommendation for surgical excision? Radiology 224:548 554

24. Schueller G, Jaromi S, Ponhold L, Fuchsjaeger M, Memarsadeghi M, Rudas M, Weber M, Liberman L, Helbich TH (2008) USguided 14-gauge core-needle breast biopsy: results of a validation study in 1352 cases. Radiology 248:406-413

25. Youk JH, Kim EK, Kim MJ, Oh KK (2008) Sonographically guided 14-gauge core needle biopsy of breast masses: a review of 2, 420 cases with long-term follow-up. AJR Am J Roentgenol 190:202-207

26. Jang M, Cho N, Moon WK, Park JS, Seong MH, Park IA (2008) Underestimation of atypical ductal hyperplasia at sonographically guided core biopsy of the breast. AJR Am J Roentgenol 191:1347-1351

27. Eby PR, Ochsner JE, DeMartini WB, Allison KH, Peacock S, Lehman CD (2009) Frequency and upgrade rates of atypical ductal hyperplasia diagnosed at stereotactic vacuum-assisted breast biopsy: 9-versus 11-gauge. AJR Am J Roentgenol 192:229-234

28. Youk JH, Kim EK, Kim MJ (2009) Atypical ductal hyperplasia diagnosed at sonographically guided 14-gauge core needle biopsy of breast mass. AJR Am J Roentgenol 192:1135-1141

29. Liberman L, Holland AE, Marjan D, Murray MP, Bartella L, Morris EA, Dershaw DD, Wynn RT (2007) Underestimation of atypical ductal hyperplasia at MRI-guided 9-gauge vacuumassisted breast biopsy. AJR Am J Roentgenol 188:684-690

30. American College of Radiology. ACR BI-RADS: magnetic resonance imaging. In: ACR Breast Imaging Reporting and Data System (BI-RADS) breast imaging atlas. Reston, VA: American College of Radiology, 2003

31. Noroozian M, Gombos EC, Chikarmane S, Georgian-Smith D, Raza S, Denison CM, Frost EP, Birdwell RL (2010) Factors that impact the duration of MRI-guided core needle biopsy. AJR Am J Roentgenol 194:W150-W157 
32. Malhaire C, El Khoury C, Thibault F, Athanasiou A, Petrow P, Ollivier L, Tardivon A (2010) Vacuum-assisted biopsies under MR guidance: results of 72 procedures. Eur Radiol 20:15541562

33. Cohen MA (2004) Cancer upgrades at excisional biopsy after diagnosis of atypical lobular hyperplasia or lobular carcinoma in situ at core needle biopsy: some reasons why. Radiology 231:617-621

34. Brem RF, Lechner MC, Jackman RJ, Rapelyea JA, Evans WP, Philpotts LE, Hargreaves J, Wasden S (2008) Lobular neoplasia at percutaneous breast biopsy: variables associated with carcinoma at surgical excision. AJR Am J Roentgenol 190:637-641

35. Liberman L, Mason G, Morris EA, Dershaw DD (2006) Does size matter? Positive predictive value of MRI-detected breast lesions as a function of lesion size. AJR Am J Roentgenol $186: 426-430$

36. Gutierrez RL, Demartini WB, Eby P, Kurland BF, Peacock S, Lehman CD (2009) Clinical indication and patient age predict likelihood of malignancy in suspicious breast MRI lesions. Acad Radiol 16:1281-1285

37. Houssami N, Ciatto S, Ellis I, Ambrogetti D (2007) Underestimation of malignancy of breast core-needle biopsy: concepts and precise overall and category-specific estimates. Cancer 109:487-495

38. O’Malley FP, Mohsin SK, Badve S, Bose S, Collins LC, Ennis M, Kleer CG, Pinder SE, Schnitt SJ (2006) Interobserver reproducibility in the diagnosis of flat epithelial atypia of the breast. Mod Pathol 19:172-179 